\title{
A NONSOLVABLE COMPLEX VECTOR FIELD WITH HÖLDER COEFFICIENTS
}

\author{
HOWARD JACOBOWITZ
}

(Communicated by Barbara L. Keyfitz)

ABSTRACT. It is known that the equation

$$
\frac{\partial u}{\partial t}-\alpha(\xi, t) \frac{\partial u}{\partial \xi}=f(\xi, \tau)
$$

is solvable in a neighborhood of the origin provided $\operatorname{Im} \alpha$ does not change sign and $\alpha$ is at least Lipschitz smooth. An example is given where solvability fails although $\alpha$ is of Hölder class $\lambda$ for all $0<\lambda<1$. Further, the only solutions to

$$
\frac{\partial u}{\partial t}-\alpha(\xi, t) \frac{\partial u}{\partial \xi}=0
$$

are the constant functions.

Consider the partial differential operator with a complex coefficient

$$
L=\frac{\partial}{\partial t}-\alpha(\xi, t) \frac{\partial}{\partial \xi} .
$$

It is well known that if $\alpha$ is $C^{\infty}$ and $\operatorname{Im} \alpha$ does not change sign in a neighbourhood of the origin then $L$ is solvable near the origin. In fact, several of the usual proofs work if $\alpha(x, y)$ is only $C^{2}$. Recently, Hounie established solvability even in the case where $\alpha$ is only Lipschitz. We give an example to show this smoothness is optimal. Namely, we find a function $\alpha$ such that $\operatorname{Im} \alpha(x, y)$ has an isolated zero at the origin and $\alpha$ is of Hölder class $C^{\lambda}$ for all $0<\lambda<1$, and yet $L$ is not solvable at the origin and, indeed, $L$ is not even homogeneously solvable there. This example, in part, motivated the work in [Ho]. The author thanks J. Hounie and F. Treves for several interesting conversations and the referee for his objection to an embarrassingly indirect proof of the corollary to Theorem 1. The referee also pointed out that an analogous difference between Hölder and Lipschitz occurs for uniqueness in the Cauchy problem for strictly hyperbolic operators [CJS].

Received by the editors June 25, 1990 and, in revised form, April 11, 1991; presented at the Special Session on Applications of Microlocal Analysis to Partial Differential Equations, AMS Eastern Section, October 12, 1991.

1991 Mathematics Subject Classification. Primary 35F99.

This work was partially supported by NSF Grant 8803086 . 


\section{SOlVABILITY FOR LIPSCHITZ COEFFICIENTS}

We consider the partial differential operator

$$
L u=u_{t}-\alpha(x, t) u_{x}+\gamma(x, t) u
$$

and the domain

$$
\Omega_{\varepsilon}\{(x, t):-\infty<x<\infty,-\varepsilon<t<\varepsilon\} .
$$

Here is the result of Hounie:

Theorem 1. Let $\alpha(x, t)$ be Lipschitz on $\mathbf{R}^{2}$ and $\gamma(x, t)$ measurable and bounded on $\mathbf{R}^{2}$. If $\operatorname{Im} \alpha \geq 0$ then there exists some $\varepsilon>0$ such that for each $f \in L^{2}\left(\Omega_{\varepsilon}\right)$, there exists some $u \in L^{2}\left(\Omega_{\varepsilon}\right)$ satisfying $L u=f$ in the sense of distributions.

Remark. The Theorem holds just as well for $\operatorname{Im} \alpha \leq 0$, as can be seen by replacing $x$ by $-x$. It also holds for $L$ replaced by its transpose,

$$
L^{t} u=-u_{t}+\alpha u_{x}+\left(\gamma+\alpha_{x}\right) u \text {. }
$$

To prove the theorem (for the transpose), it is enough to prove the $L^{2}$ inequality

$$
\|u\| \leq C\|L u\| \text { for all } u \in C_{0}^{\infty}\left(\Omega_{\varepsilon}\right) .
$$

We sketch Hounie's proof. Let $\alpha(x, t)=a(x, t)+i b(x, t)$. We start with the identity

$$
\begin{aligned}
2 \operatorname{Re} \iint(1-t)(L u) \bar{u} d x d t= & 2\|u\|^{2}+\iint(1-t) a_{x}|u|^{2} d x d t \\
& +2 \operatorname{Re} \iint(1-t) b(D u) \bar{u} d x d t \\
& +2 \operatorname{Re} \iint(1-t) \gamma|u|^{2} d x d t
\end{aligned}
$$

where $D=-i \partial / \partial x$, and then easily derive

$$
\|L u\|\|u\| \geq\|u\|^{2}+2 \operatorname{Re} \iint(1-t) b D u \bar{u} d x d t
$$

provided $\left\|a_{x}\right\|_{\infty}$ and $\mid \gamma \|_{\infty}$ are small (as they are after a suitable change of variables). Speaking roughly, if $b \geq 0$ were to commute with the 'square root' of $D$, then the last term would be nonnegative and hence negligible. So the estimate reduces to the commutability up to an operator of order zero of a Lipschitz function and an operator of order one-half. Still speaking roughly, this is what Hounie establishes. The analogous result was known for a Lipschitz function and a singular integral operator of order zero [Ca, CM]. Note that the condition $\operatorname{Im} \alpha \geq 0$ implies the classical condition $P$ of Nirenberg-Treves [NT]. Hounie shows that, assuming condition $P$,

$$
L=\frac{\partial}{\partial t}-i \sum_{j=1}^{n} b_{j}(x, t) \frac{\partial}{\partial x_{j}}+c(x, t)
$$

is solvable if each $b_{j}$ is real and Lipschitz and $c$ is bounded and measurable. But if $b_{j}$ is not assumed to be real then for smoothness one must assume each 
$\partial b_{j} / \partial x_{k}$ is Lipschitz. However, as we have outlined (and as is easily seen from Hounie's proof), for $\operatorname{Im} \alpha \geq 0$ in place of Condition $P$ and for $n=1$, this extra smoothness is not necessary.

To show that this theorem is sharp in a neighborhood of a point, with respect to the smoothness of $\alpha$, one would need to find some $\alpha$ and $f$ with

$$
f \in L^{2}, \quad \alpha \in C^{\lambda} \text { all } \lambda, \quad 0<\lambda<1, \quad \alpha \notin \operatorname{Lip}, \quad \operatorname{Im} \alpha \geq 0
$$

for which

$$
u_{t}-\alpha u_{x}=f
$$

has no solution $u \in L^{2}$ in any neighborhood of the origin. This would lead to the problem of explaining what (1) means, since the distribution $u_{x}$ usually cannot be multiplied by the nonsmooth function $\alpha$. Rather than deal with this, we slightly strengthen Hounie's result and then establish sharpness for this strengthened result. We use $H^{1}(\Omega)$ to denote the Sobolev space of functions $u$ in $L^{2}$, with first derivates also in $L^{2}$.

Corollary. Let $\alpha(x, t)$ be Lipschitz on $\mathbf{R}^{2}$ and let $\operatorname{Im} \alpha \geq 0$. There exists some $\varepsilon>0$ and some neighborhood of the origin $\Omega^{\prime} \subset \Omega_{\varepsilon}$ such that for every $f \in H^{1}\left(\Omega_{\varepsilon}\right)$ there exists some $u \in H^{1}\left(\Omega^{\prime}\right)$ that satisfies $u_{t}-\alpha(x, t) u_{x}=f$.

Proof. A formal proof of this is easy. If we knew that $v=u_{x}$ was in $L^{2}$, then it would satisfy the equation

$$
v_{t}-\alpha v_{x}-\alpha_{x} v=g
$$

with $g=f_{x}$. So instead we start with (2). By the theorem, this equation has some solution $v(x, t)$ in $L^{2}(\Omega)$. We clearly may take $\alpha$ and $f$ to be compactly supported, since we seek only a local result. Let $\chi(x)$ be a compactly supported smooth function that is equal to one on the projection of $\operatorname{supp} \alpha \cup \operatorname{supp} f$ to the $x$-axis and set

$$
V=\chi v .
$$

Thus

$$
V_{t}-\alpha V_{x}-\alpha_{x} V=g .
$$

Let $H$ be the Heaviside function

$$
H(x)= \begin{cases}0 & \text { for } x<0 \\ 1 & \text { for } x>0\end{cases}
$$

and set

$$
U(x, t)=H * V(x, t)=\int_{-\infty}^{\infty} H(x-y) V(y, t) d y .
$$

It follows that

$$
U \in L^{2}, \quad U_{x}=V, \quad U_{t}=H * V_{t} .
$$

Thus

$$
\begin{aligned}
U_{t} & =H *\left(g+\alpha V_{x}+\alpha_{x} V\right) \\
& =H *\left(f_{x}+(\alpha V)_{x}\right)=f+\alpha V=f+\alpha U_{x} .
\end{aligned}
$$

So $U_{t}-\alpha U_{x}=f$ and $U \in H^{1}$.

Our goal is to show that this Corollary is sharp. 
Theorem 2. There exist functions $\alpha(x, t)$ and $f(x, t)$ with

(1) $\alpha$ in $C^{\lambda}\left(\mathbf{R}^{2}\right)$ for every $\lambda, 0<\lambda<1$;

(2) $\operatorname{Im} \alpha>0$ except at the origin;

(3) $f \in C^{\infty}\left(\mathbf{R}^{2}\right)$;

but such that the equation $u_{t}-\alpha(x, t) u_{x}=f$ has no solution $u \in H^{1}(B)$ where $B$ is any arbitrarily small neighborhood of the origin.

Remark. This leaves open the question of whether every $L=\partial / \partial x$ $-i b(x, y) \partial / \partial y$, with $b$ real, nonnegative, and of class $C^{\lambda}$ is solvable. Also, one could look for a sharper result by considering function spaces lying between $\bigcap_{\lambda<1} C^{\lambda}$ and Lipschitz. For example, there is the 'Hölder one' space $\Lambda_{1}$ from harmonic analysis (see [St]). The coefficient in our example is not in $\Lambda_{1}$ and so Theorem 1 might continue to hold for $\alpha(x, t)$ in this less restrictive class.

\section{THE EXAMPLE}

Define the sets

$$
X=\left\{(x, y) \in \mathbf{R}^{2}:-1 \leq x \leq 1, y=0\right\}
$$

and

$$
D=\left\{(x, y) \in \mathbf{R}^{2}: x^{2}+y^{2}<2\right\} .
$$

Let $\phi(x)$ and $\psi(y)$ be the $C^{\infty}$ functions

$$
\phi(x)= \begin{cases}e^{-1 /(x-1)^{2}}, & x>1, \\ 0, & -1 \leq x \leq 1, \\ e^{-1 /(x+1)^{2}}, & x<-1\end{cases}
$$

and

$$
\psi(y)= \begin{cases}e^{-1 / y^{2}}, & y \neq 0, \\ 0, & y=0 .\end{cases}
$$

Define a transformation $F: \mathbf{R}^{2} \rightarrow \mathbf{R}^{2}$ by

$$
\xi=(\psi(y)+\phi(x)) x, \quad \eta=y .
$$

Clearly there is some deleted neighborhood $N$ of the origin for which the restriction $F: D-X \rightarrow N$ is a $C^{\infty}$ diffeomorphism.

Let $C^{1-}$ denote $\bigcap_{0<\lambda<1} C^{\lambda}$. So $f \in C^{1-}(\Omega)$ if for each $\lambda$ in $0<\lambda<1$, there is a constant $C_{\lambda}$ with $|f(p)-f(q)|<C_{\lambda}|p-q|^{\lambda}$ for all $p$ and $q$ in $\Omega$.

Lemma 1. The vector field $L=-2 i F_{*}(\partial / \partial \bar{z})$ is well defined on $N \cup 0$ and has the form

$$
L=\frac{\partial}{\partial \eta}-\alpha(\xi, \eta) \frac{\partial}{\partial \xi}
$$

where $\alpha$ is in $C^{1-}(N \cup 0)$ but is not in $\operatorname{Lip}(N \cup 0)$. Further, $\operatorname{Im} \alpha>0$ except at $(\xi, \eta)=(0,0)$.

Proof. We have

$$
\begin{aligned}
2 F_{*}\left(\frac{\partial}{\partial \bar{z}}\right) & =F_{*}\left(\frac{\partial}{\partial x}\right)+i F_{*}\left(\frac{\partial}{\partial y}\right) \\
& =i\left(\frac{\partial}{\partial \eta}+\psi^{\prime}(y) x \frac{\partial}{\partial \xi}\right)+\left(\psi(y)+\phi(x)+\xi \phi^{\prime}(x)\right) \frac{\partial}{\partial \xi}
\end{aligned}
$$


and on $N, F^{-1}$ may be used to express $x$ and $y$ as functions of $\xi$ and $\eta$, while at the origin of the $(\xi, \eta)$-plane

$$
2 F_{*}\left(\frac{\partial}{\partial \bar{z}}\right)=i \frac{\partial}{\partial \eta}
$$

Thus

$$
L=\frac{\partial}{\partial \eta}+\left(x \psi^{\prime}(y)-i\left(\psi(y)+\phi(x)+x \phi^{\prime}(x)\right)\right) \frac{\partial}{\partial \xi}
$$

is well defined and continuous on $N \cup 0$. We have

$$
\alpha(\xi, \eta)=-x \psi^{\prime}(y)+i\left(\psi(y)+\phi(x)+x \phi^{\prime}(x)\right)
$$

and $\operatorname{Im} \alpha$ is zero only when $(x, y) \in X$. Thus, as a function of $\xi$ and $\eta$, $\operatorname{Im} \alpha$ is zero only at the origin and is positive elsewhere. If we differentiate, with respect to $\xi$, the first defining function for $F$, we obtain, away from the origin in the $(\xi, \eta)$ plane,

$$
1=\left(\psi(\eta)+\phi(x)+x \phi^{\prime}(x)\right) x_{\xi} .
$$

Thus, for any fixed $\eta \neq 0,1=\psi(\eta) x_{\xi}(0, \eta)$. This implies that $\lim _{\eta \rightarrow 0} \psi^{\prime}(\eta) x_{\xi}(0, \eta)=\infty$ and so the coefficient of $L$ is not differentiable at the origin.

In the next section, we show that this coefficient is actually in $C^{1-}$ but not in Lip. We now show that at the origin $L$ is neither solvable nor homogeneously solvable in the $C^{1}$-sense. Then we strengthen this to obtain Theorem 2 .

Lemma 2. There exists some $C^{\infty}$ function $f$ for which there is no $C^{1}$ function $u$ that satisfies $L u=f$ in a neighborhood of the origin.

Proof. We use the argument introduced by Grusin [Gr]. See also [Ni]. Let $B_{j}$ be a disjoint set of balls in the upper half plane in $(x, y)$ space that converge to 0 . Let $D_{j}=F\left(B_{j}\right)$ and choose some $C^{\infty}$ function $f$ that is positive in each $D_{j}$ and zero outside of $\bigcup_{j=1}^{\infty} D_{j}$. (The simplest way to do this would be to take such a function $f_{1}(x, y)$ for $\left\{B_{j}\right\}$, set $f_{2}(\xi, \eta)=f_{1}\left(F^{-1}(\xi, \eta)\right)$ for $(\xi, \eta) \in \sup D_{j}$ and $f_{2}(\xi, \eta)=0$ otherwise, and then set $f(\xi, \eta)=\lambda(\xi, \eta) f_{2}(\xi, \eta)$ where $\lambda$ is positive outside of the origin and is chosen to go to zero fast enough at the origin so as to make $f(\xi, \eta)$ of class $C^{\infty}$.) Assume that $u$ is a $C^{1}$ function that satisfies $L u=f$ in some neighborhood of the origin. Then for $U=u \circ F$ and $G=f \circ F$ we have $U_{\bar{z}}=G$ in some neighborhood $\Omega$ of $X$. So $U$ is holomorphic on $\Omega-\cup B_{j}$ and is a constant on $X$. Thus $U$ is a constant on $\Omega-\cup B_{j}$ and so

$$
\int_{\partial B_{j}} U d z=U \int_{\partial B_{j}} d z=0
$$

but

$$
\int_{\partial B_{j}} U d z=\iint_{B_{j}} U_{\bar{z}} d \bar{z} d z=\iint_{B_{j}} G d \bar{z} d z \neq 0 .
$$

This contradiction shows that there is no solution $u \in C^{1}$ to $L u=f$.

We now show that even the homogeneous equation $L u=0$ is nonsolvable, in the sense that the only solutions are the constant functions. The first example of such a nonhomogeneously solvable operator, which even had smooth coefficients, was given by Nirenberg [Ni]. 
Lemma 3. Let $O \subset N \cup\{0\}$ be a connected neighborhood of the origin. If $h \in C^{1}(O)$ satisfies $L h=0$ in $O$, then $h$ is identically a constant in $O$.

Proof. The function $H=h \circ F$ is holomorphic on $F^{-1}(O)$ and constant on $X$. Thus $H$ is a constant and so $h$ is also a constant.

Next we show that $L u=f$ also fails to have $H^{1}$ solutions in any neighborhood of the origin. This is the proof of Theorem 2 since the value of the functions $\alpha(x, t)$ and $f(x, t)$ outside some neighborhood of the origin are clearly irrelevant.

We start with a change of variables formula, written in complex notation.

Lemma 4. Let $\Phi: \mathscr{D}_{1} \rightarrow \mathscr{D}_{2}$ be a diffeomorphism of domains in $\mathrm{C}$ and let $u \in H^{1}\left(\mathscr{D}_{2}\right)$. Let $\zeta=\Phi(z, \bar{z}), U=u \circ \Phi$, and

$$
w=\left(\left|\Phi_{z}\right|^{2}+\left|\Phi_{\bar{z}}\right|^{2}\right) /\left(\left|\Phi_{z}\right|^{2}-\left|\Phi_{\bar{z}}\right|^{2}\right) .
$$

If $U$ is holomorphic on $\mathscr{D}_{1}$ then $\left|U_{z}\right|^{2} w$ is integrable over $\mathscr{D}_{1}$ and

$$
\iint_{\mathscr{D}_{1}}\left|U_{z}\right|^{2} w(z, \bar{z}) d z d \bar{z}=\iint_{\mathscr{D}_{2}}\left(\left|u_{\zeta}\right|^{2}+\left|u_{\bar{\zeta}}\right|^{2}\right) d \zeta d \bar{\zeta}
$$

Proof. The proof is immediate since

$$
d \zeta d \bar{\zeta}=\left(\left|\Phi_{z}\right|^{2}-\left|\Phi_{\bar{z}}\right|^{2}\right) d z d \bar{z}
$$

and

$$
\left|u_{\zeta}\right|^{2}+\left|u_{\bar{\zeta}}\right|^{2}=\frac{\left|\Phi_{z}\right|^{2}+\left|\Phi_{\bar{z}}\right|^{2}}{\left(\left|\Phi_{z}\right|^{2}-\left|\Phi_{\bar{z}}\right|^{2}\right)^{2}}\left|U_{z}\right|^{2}
$$

We will be interested in the map $F$ when $x$ is close to zero, so $\xi=\psi(y) x$, $\eta=y$ with $\psi$ as in the previous section. Then

$$
w(x, y)=\left\{1+\psi^{2}+\left(\psi^{\prime}\right)^{2} x^{2}\right\} /(2 \psi) .
$$

Note that $w>c_{N} y^{-N}$ for $y$ small and positive. In particular,

$$
\lim \frac{1}{r^{4}} \iint_{B_{i}} \frac{d x d y}{w(x, y)}=0
$$

for any sequence of balls $B_{i}=B\left(z_{i}, r_{i}\right)$ where $0<\operatorname{Im} z_{i} \rightarrow 0$ and $r_{i}=\frac{1}{2} \operatorname{Im} z_{i}$. Lemma 5. Let $w(x, y)$ be any function satisfying (10). If $V(z)$ is holomorphic in some strip $a<x<b, 0<y<\varepsilon$, and

$$
\int_{a}^{b} \int_{0}^{\varepsilon}|V(z)|^{2} w(z, \bar{z}) d z d \bar{z}
$$

is bounded, then $V$ is identically zero.

Proof. Choose any $z$ for which $B(z, r)$ is in the strip, for $r=\frac{1}{2} \operatorname{Im} z$. So

$$
\begin{aligned}
|V(z)| & \leq \frac{1}{\pi r^{2}} \iint_{B(z, r)}|V(z)| d x d y \\
& \leq \frac{1}{\pi r^{2}}\left(\iint|V(z)|^{2} w(z, \bar{z}) d x d y\right)^{1 / 2}\left(\iint \frac{1}{w(z, \bar{z})} d x d y\right)^{1 / 2} .
\end{aligned}
$$

Thus, $V(z)$ approaches zero as $\operatorname{Im} z \rightarrow 0$. Hence $V \equiv 0$.

Now let $f$ be as in the proof of Lemma 2 and assume that there is some neighborhood of the origin, $\Omega$, and some $u \in H^{1}(\Omega)$, satisfying $L u=f$ on 
$\Omega$. Again, set $U=u \circ F$. This certainly makes sense on $D-X$. Note also that $U$ is smooth on $D-X$ since it satisfies $-2 i U_{\bar{z}}=f \circ F$. But, as a consequence of Lemma 4, on this set, $\iint\left|U_{z}\right|^{2} w(z, \bar{z}) d x d y$ is bounded, for $w$ given by (9). Thus the hypothesis of Lemma 5 applies to $V=U_{z}$ (or at least it does for $0>y>-\varepsilon$, since we have that $V$ is holomorphic outside of the support of $f$ ). It follows that $U$ is a constant outside of the support $f$. Just as before, this leads to a contradiction. This concludes the proof of Theorem 2.

Remark. It is clear from these proofs that Lemma 3 holds also for $h \in H^{1}(O)$.

\section{THE COEFFICIENT OF $L$}

We want to show that $\alpha(\xi, \eta)$ is in $C^{1-}$ but not in Lip. We will sketch the proof of a somewhat more precise result. We use the notation $C^{\lambda}(\xi) \times \operatorname{Lip}(\eta)$ to denote functions that for fixed $\eta$ are of class $C^{\lambda}$ with norm independent of $\eta$, for fixed $\xi$ are of class Lip with norm independent of $\xi$, and similarly for other function spaces. Note that $C^{\lambda}(\xi, \eta)=C^{\lambda}(\xi) \times C^{\lambda}(\eta)$ and $\operatorname{Lip}(\xi, \eta)=$ $\operatorname{Lip}(\xi) \times \operatorname{Lip}(\eta)$.

The following smoothness results are valid for each $\lambda<1$.

(a) $\psi^{\prime}(\eta) x(\xi, \eta)$ is in $C^{\lambda}(\xi) \times C^{\infty}(\eta)$ but is not in $\operatorname{Lip}(\xi)$.

(b) $\phi(x(\xi, \eta))$ is in $\operatorname{Lip}(\xi) \times C^{1}(\eta)$ but is not in $C^{1}(\xi)$.

(c) $\phi^{\prime}(x(\xi, \eta)) x(\xi, \eta)$ is in $C^{\lambda}(\xi, \eta)$ but is not in $\operatorname{Lip}(\xi)$ and is not in $\operatorname{Lip}(\eta)$.

We start with

$$
\xi=(\psi(\eta)+\phi(x(\xi, \eta))) x(\xi, \eta) .
$$

The properties of $\phi$ and $\psi$ imply that

$$
|\xi-\bar{\xi}| \geq \psi(\eta)|x-\bar{x}|
$$

and

$$
|\xi-\bar{\xi}| \geq|\phi-\bar{\phi}|
$$

where $\bar{x}=x(\bar{\xi}, \eta)$ and $\bar{\phi}=\phi(\bar{x})$. (Here the bar does not mean conjugation.) Thus, for $\eta \neq 0$,

$$
\frac{\left|\psi^{\prime}(\eta) x-\psi^{\prime}(\eta) \bar{x}\right|}{|\xi-\bar{\xi}|^{\lambda}} \leq \frac{\left|\psi^{\prime}(\eta)\right|}{\psi(\eta)^{\lambda}}=\frac{2}{|\eta|^{3}} e^{(1-\lambda) / \eta^{2}} .
$$

So $\psi^{\prime}(\eta) x(\xi, \eta) \in C^{\lambda}(\xi)$. If $\xi$ and $\bar{\xi}$ are small enough, $\phi$ and $\bar{\phi}$ are zero; so in this case, and for $\lambda=1,(14)$ becomes an equality. Hence $\psi^{\prime}(\eta) x(\xi, \eta) \notin$ $\operatorname{Lip}(\xi)$.

If we differentiate (11) with respect to $\eta$ we obtain

$$
0=\psi^{\prime}(\eta) x+\left(\psi+\phi+x \phi^{\prime}\right) x_{\eta},
$$

and so $\left|x_{\eta}\right|<\left|\psi^{\prime}(\eta) x / \psi(\eta)\right|<C / \eta^{3}$. Repeated differentiation and a simple induction establish that $\left|D_{\eta}^{n} x\right| \leq c_{n} /|\eta|^{3 n}$ and thus that $\psi^{\prime}(\eta) x(\xi, \eta) \in C^{\infty}(\eta)$. This proves (a). We have directly from (13) that $\phi \in \operatorname{Lip}(\xi)$. To see that this function is not in $C^{1}(\xi)$ it suffices to take $\eta=0$ and $\xi= \pm \varepsilon$, where $\varepsilon$ is a 
small positive quantity. Then $\xi=\phi(x) x$ and $x= \pm(1+\delta)$ where $\delta$ is also a small positive quantity. Thus

$$
\lim _{\varepsilon \rightarrow 0} \frac{\phi(x(\xi, 0))}{\xi}= \pm 1
$$

and $\phi(x) \notin C^{1}(\xi)$. Since $\phi_{\eta}$ exists everywhere, except possibly at the origin, and satisfies

$$
\left|\phi_{\eta}\right|=\left|\phi^{\prime}(x) x_{\eta}\right| \leq\left|\phi^{\prime}(x)\right| \frac{\left|\psi^{\prime}(\eta)\right|}{\left|\phi^{\prime}(x)\right|}=\left|\psi^{\prime}(\eta)\right|,
$$

we need only show that $\phi_{\eta}$ exists and is zero at the origin. But $\phi(x(0, \eta))=0$, so $\phi_{\eta}(0,0)=0$. This proves $(\mathrm{b})$.

For (c), we first let $\bar{x}=x(\bar{\xi}, \eta)$ and bound $\left|x \phi^{\prime}-\bar{x} \bar{\phi}^{\prime}\right| /|\xi-\bar{\xi}|^{\lambda}$. If $\phi=\bar{\phi}$ then also $x \phi^{\prime}=\bar{x} \bar{\phi}^{\prime}$, and there is nothing to do. So we assume $\phi \neq \bar{\phi}$ and we use (13) to write

$$
\frac{\left|x \phi^{\prime}-\bar{x} \bar{\phi}^{\prime}\right|}{|\xi-\bar{\xi}|^{\lambda}} \leq \frac{\left|x \phi^{\prime}-\bar{x} \bar{\phi}^{\prime}\right|}{|\phi-\bar{\phi}|^{\lambda}}
$$

If $\bar{\phi}=0$, then for the right-hand side we have

$$
\frac{\left|x \phi^{\prime}\right|}{|\phi|^{\lambda}} \leq 2\left|\frac{x}{(x \pm 1)^{3}}\right| e^{-(1-\lambda) /(x \pm 1)^{2}}<B
$$

provided $\lambda<1$. (For $|x|>1$ and $\lambda \leq 1$ the left sign is an equality. This shows that $x \phi^{\prime} \notin \operatorname{Lip}(\xi)$.) Thus in addition to $\phi \neq \bar{\phi}$ we may assume that neither is zero. That is, we now take $|x|>1$ and $|\bar{x}|>1$. Let $\tau=\phi(x(\xi, \eta))$. Solving for $x$ and then substituting, we obtain

$$
x \phi^{\prime}(x)=2\left(1+(-\ln \tau)^{-1 / 2}\right)(-\ln \tau)^{3 / 2} \tau .
$$

In light of (15), our goal is to show that this function of $\tau$ is in $C^{\lambda}$ but is not Lipschitz. We skip the simple verification of this. So $x \phi^{\prime}(x) \in C^{\lambda}(\xi)$ but is not in $\operatorname{Lip}(\xi)$. It is much the same for $x \phi^{\prime}(x)$ as a function of $\eta$. We write

$$
\frac{\left|x \phi^{\prime}-\bar{x} \bar{\phi}^{\prime}\right|}{|\eta-\bar{\eta}|^{\lambda}}=\frac{\left|x \phi^{\prime}-\bar{x} \bar{\phi}^{\prime}\right|}{|\phi-\bar{\phi}|^{\lambda}} \frac{|\phi-\bar{\phi}|^{\lambda}}{|\eta-\bar{\eta}|^{\lambda}}
$$

and use that $x \phi^{\prime}$ is $C^{\lambda}$ in $\phi$ (this is what was just proved) and that $\phi$ is $C^{1}$ in $\eta$. To see that $x \phi^{\prime} \notin \operatorname{Lip}(\eta)$, we look at (16) for $\lambda=1$. We know that $\phi \in C^{1}(\eta)$ and $x \phi^{\prime} \notin \operatorname{Lip}(\phi)$. Thus $x \phi^{\prime} \notin \operatorname{Lip}(\eta)$. This proves (c). The only remaining term in the coefficient of $L$ is $\psi(\eta)$, which of course is smooth. So we are done.

Remark. It is easy to see even without calculating that $\psi^{\prime}(\eta) x(\xi, \eta)$ is not in $\operatorname{Lip}(\xi, \eta)$. For the vector field $F_{*} \partial / \partial y$ has as integral curves through the origin all the curves $t \rightarrow F\left(x_{0}, t\right)$ for each $x_{0}$ with $\left|x_{0}\right|<1$. But a vector field that is Lipschitz has a unique integral curve through each point.

\section{REFERENCES}

[Ca] A. P. Calderón, Commutators of singular integral operators, Proc. Nat. Acad. Sci. U.S.A. 53 (1965), 1092-1099.

[CJS] F. Colombini, E. Jannelli, and S. Spagnolo, Non uniqueness in hyperbolic Cauchy problems, Ann. of Math. (2) 126 (1987), 495-524. 
[CM] R. Coiffman et Y. Meyer, Au delà des opérateurs pseudo-différentiels, Astérisque 57 (1979).

[Gr] V. Grusin, A certain example of a differential equation without solutions, Mat. Z. 10 (1971), 125-128; Math. Notes 10 (1971), 499-501.

[Ho] J. Hounie, Local solvability of first order linear operators with Lipschitz coefficients, Duke Math. J. 62 (1991), 467-477.

[Ni] L. Nirenberg, Lectures on linear partial differential equations, Amer. Math. Soc., Providence, RI, 1973.

[NT] L. Nirenberg and F. Treves, Solvability of a first order linear partial differential equation, Comm. Pure Appl. Math. 16 (1963), 331-351.

[St] E. Stein, Singular integrals and the differentiability properties of functions, Princeton, NJ, 1970.

Mathematical Sciences Department, Rutgers University, Camden, New Jersey, 08102 E-mail address: jacob@math.ias.edu 